\title{
Mongolian Medical Acupuncture Improves Sleep Quality in Patients with Primary Insomnia
}

\author{
Lengge $\mathrm{Si}^{1}$, Lidao Bao ${ }^{2}$, Rui Peng ${ }^{2}$, Yuehong $\mathrm{Wang}^{3}$, Agula $\mathrm{B}^{1,3 \text {, * }}$ \\ ${ }^{1}$ School of Preclinical Medicine, Beijing University of Chinese Medicine, Beijing, China \\ ${ }^{2}$ Department of Pharmacy, Affiliated Hospital of Inner Mongolia Medical University, Hohhot, China \\ ${ }^{3}$ College of Mongolian Medicine, Inner Mongolia Medical University, Hohhot, China
}

\section{Email address:}

349881704@qq.com (Lengge Si), baolidao237@qq.com (Lidao Bao), agulab@163.com (Agula B)

\section{To cite this article:}

Lengge Si, Lidao Bao, Rui Peng, Yuehong Wang, Agula B. Mongolian Medical Acupuncture Improves Sleep Quality in Patients with Primary Insomnia. American Journal of Clinical and Experimental Medicine. Vol. 3, No. 6, 2015, pp. 372-377. doi: 10.11648/j.ajcem.20150306.19

\begin{abstract}
The aim of this study was to evaluate the therapeutic effects of the Mongolian medical warm needle acupuncture (MMA) in treating patients with primary insomnia. 40 patients with primary insomnia were randomly divided into 2 groups as (1) Control Group, who received automatic neural balance regulation (ANBR), and (2) MMA Group, who received ANBR plus MMA treatment. The MMA treatment was administered to 5 acupuncture points according to traditional Mongolian medicine. Pittsburgh Sleep Quality Index (PSQI) was used to quantitatively measure patients' outcome at time 0 (prior to study involvement), time 1 (after 8-week treatment), and time 2 (follow-up examination 4-week post-treatment). Multivariate analyses were conducted using treatment, gender, time, and age as factors and covariates. Cronbach's alpha coefficient was used to evaluate internal homogeneity. MMA significantly reduced PSQI in insomniac patients compared with control $(t=9.59, p<$ 0.001). Six component scores of the PSQI were internally consistent (Cronbach's alpha coefficient $=0.89$ ). Out of the 6 components of PSQI, MMA significantly improved subjective sleep quality, sleep latency, and daytime dysfunction. The Mongolian medical warm needle acupuncture combined with automatic neural balance regulation has significant therapeutic effects in treating primary insomnia.
\end{abstract}

Keywords: Mongolian Medical Warm Needle Acupuncture, Primary Insomnia, Automatic Neural Balance Regulation

\section{Introduction}

Insomnia causes daytime functional defects in patients, decreases health-related quality of life, and lead severe negative effects to individuals and society [1]. Generally, insomnia, both a symptom and diagnosis, is state of dissatisfaction with the sleep quality including difficulty falling asleep, difficulty maintaining sleep, frequent awakenings, dreaminess, early awakening, difficulty returning to sleep after awakenings, discomfort after awakening, fatigue, or daytime tiredness [2]. Previous researches across the world showed that an average of $17 \%$ of the global population suffered from insomnia [3, 4].

Previous researches have demonstrated that the acupuncture exerts significant therapeutic effects on insomnia and also has advantages over sleeping medicine with respects to safety, reliability, convenience [5]. Similar to the acupuncture of Traditional Chinese Medicine, Mongolian medical acupuncture (MMA) utilizes specially-made warming silver needles to stimulate specific sites (acupoints by the acupuncture nomenclature) of body for the prevention and management of diseases $[6,7]$. Currently, there have been a number of studies on treating insomnia with the MMA [8]; however, there are few studies that quantitatively evaluate the effects of MMA therapy.

This research aimed at quantifying the effects of treatment of insomnia with the MMA in patients with primary insomnia, with the effects of treatment evaluated by the self-rated Pittsburgh Sleep Quality Index (PSQI) [9].

\section{Methods}

\subsection{Study Subjects}

This study was approved by the Ethics Committee of Inner Mongolia Medical University. The study site was the Department of Traditional Mongolian Medicine of Affiliated Hospital (a tertiary teaching hospital) of Inner Mongolia Medical University. The study subjects were outpatients who 
were seeking medical treatment for their medical conditions including insomnia in the Departments of Psychiatry and Internal Medicine. The patients in the two departments were referred to the Department of Traditional Mongolian Medicine for voluntary participation in this study if the patients were diagnosed with primary insomnia, which was based on the Chinese Classification of Mental Disorders Third Edition (CCMD III) issued by the Chinese Society of Psychiatry [10]. The diagnosis criteria for primary insomnia defined by CCMD III are similar to the DSM-IV-TR Diagnostic Criteria for Primary Insomnia [11]. Generally, the patients included in this study had typical symptoms of insomnia dominated by sleep disorders with all other symptoms secondary to insomnia, including difficulty falling asleep, frequent awakenings, dreaminess, early morning awakenings, inability to return to sleep after awakening, discomfort after awakening, fatigue or daytime sleepiness. These sleep disorders occurred at least three times per week and continued for $>1$ mon (for the discrimination of transient from persistent insomnia) [9]. Patients, who were aged $40-60$ years and meeting the above diagnostic criteria, were included in this study based on voluntary participation. Informed consent was obtained prior to study involvement. Patients were excluded from study involvement, if they were (1) diagnosed with secondary insomnia arising from physical diseases or mental disorders; (2) diagnosed with insomnia resulting from circadian rhythm sleep disorders, and shift work; (3) during the gestation or lactation period; (4) complicated with severe primary diseases in the cardiovascular, pulmonary, hepatic, renal, and hemopoietic systems; (5) were on a medication therapy $<3$ mon prior to study involvement; or (6) diagnosed with major depression, which would not permit a 12-week medication-free study period.

\subsection{Interventions}

For patients in MMA Group, patients underwent two procedures: the preparation procedure and the MMA therapy. Firstly, the preparation procedure is a routine practice in the Department of Traditional Mongolian Medicine for patients prior to receiving MMA. Patients were instructed to lie supine in a comfortable manner when receiving automatic neural balance regulation (ANBR) as a preparation procedure using a commercial Self-generated Physiological Coherence System (SPCS) developed by Beijing Haofeng Technology. This SPCS system is developed based on and developed by theories from Heart Math[12]. The SPCS consists of two modules: (1) ANBR - which is implemented by a software system and apparatuses for physical movement; and (2) a real-time monitoring system including a monitor screen showing up patients' biological data - including blood pressure and heart rate. The preparation procedure was considered successful if the patient scored $>60$ points, which was shown by the monitor screen. The preparation procedure would continue if a patient's score was $<60$ points. Secondly, the patients in the MMA Group were instructed to lie supine when receiving MMA that was performed at the following acupoints, including (1) Dinghui Acupoint - midpoint between eyebrows;
(2) Lumen Acupoint - located 3 cun from the midpoint of the fore hair line; (3) Heyi Acupoints - midpoint of the inferior fovea of the first thoracic vertebrae, 1 cun from the point on the left, 1 cun from the point on the right; (4) Heibaiji Acupoint midpoint between both breasts; and (5) Xinshu Acupoint - 1.5 cun lateral to the lower border of the spinous process of the 5 th thoracic vertebra on the back. During the MMA treatment, moxibustion was performed by burning 3 to 5 times at the Dinghui acupoint, 3 to 5 times at the Lumen acupoint, 3 to 9 times at the three Heyi acupoints, 3 to 7 times at the Heibaiji Acupoint, and 3 to 5 times at the Xinshu acupoint.

For patients in the Control Group, The patients received only the ANBR using the SPCS system, as described as the first procedure for patients in the MMA Group.

Each treatment session lasted for about $15 \mathrm{~min}$ for the ANBR procedure and $40 \mathrm{~min}$ for the MMA treatment. The MMA therapy was delivered by acupuncturists $(>5 \mathrm{yr}$ experience). The study sessions were conducted from Monday to Saturday, with each patient receiving 8 continuous treatments once one week (with evenly-spanned time periods), as informed to patients prior to study involvement.

\subsection{Outcome Measures}

The Pittsburgh Sleep Quality Index (PSQI) (9) was used as the questionnaires which were completed by the patients prior to study involvement (time T0), after the 8-week study period (time T1), and at follow-up examination 4 weeks after T1 (time T2). The PSQI global score (scale: $0-21)$ and the scores of the 7 component scales $(0-3$ for each component scale) of the PSQI were recorded from patients' questionnaires upon completion. The patients were observed for adverse reactions during MMA therapy. Notably, out of the original seven components (subjective sleep quality, sleep latency, sleep duration, habitual sleep efficiency, sleep disturbances, use of sleeping medication, and daytime dysfunction) of the PSQI, the component of use of sleeping medication was excluded from the PSQI global score by assigning 0 to the component score for all patients, since a 12-week medication-free period was required for study involvement, as manifested in the study protocols.

\subsection{Statistical Analyses}

Statistical analyses were conducting using SAS (version 9.2) on Windows 7. Meta-data were collected by Excel prior to analysis. PROC MIXED of SAS was used as the statistical approach to identify the main effects of treatment, gender, time, and age, as well as the interaction effects between the factors. Normality and homoscedasticity were checked by residuals and predictions generated by the LSMEANS statement of PROC MIXED. The internal homogeneity of test-retest consistency were evaluated as published previously [9]. Internal homogeneity of separate items of the PSQI was assessed by Cronbach's alpha coefficients and corrected component-total correlation coefficients. Post hoc comparisons were conducted by the LSMEANS statement of PROC MIXED with Sidak adjustment. Test-retest (consistency) was 
assessed with paired $t$ test and Pearson product-moment correlation coefficients for the PSQI global score, component scores, and individual items at time $\mathrm{T} 0, \mathrm{~T} 1$, and $\mathrm{T} 2$, respectively. This was done for the entire subject pool, as well as for separate subject groups. Data were expressed by Mean \pm S.D. $P<0.05$ was considered statistically significant.

\section{Results}

\subsection{General Data}

In total, there were 18 patients in the Control Group who completed the PSQI on the occasions at T0, T1, and T2, with the male/female ratio being 10/8; 18 patients ( 9 males and 9 females) in the MWA Group completed the PSQI on the three occasions. The 4 patients who failed to complete this study in the two groups had medical conditions that required medication/physical therapy. The mean ages for patients in the Control Group were 43.4 years $(\mathrm{SD}=3.98$; range: $36.3-51.5)$ for males and 42.8 years $(\mathrm{SD}=2.45$; range: $40.0-46.4$ ) for females; the mean ages for the MMA Group were 44.5 years $(\mathrm{SD}=6.40$; range: $32.0-53.0)$ for males and 49.8 years $(\mathrm{SD}=$ 5.10; range: $42.2-59.2$ ) for females. Student's $t$ test showed that patients' ages differed significantly between the Control and MWA groups $(t=-2.37, p=0.026)$.

\subsection{Mongolian Warm-Needling Acupuncture Improved Sleep Quality}

Multivariate analyses by PROC MIXED of SAS with treatment (Control vs. MMA), gender, and time as factors as

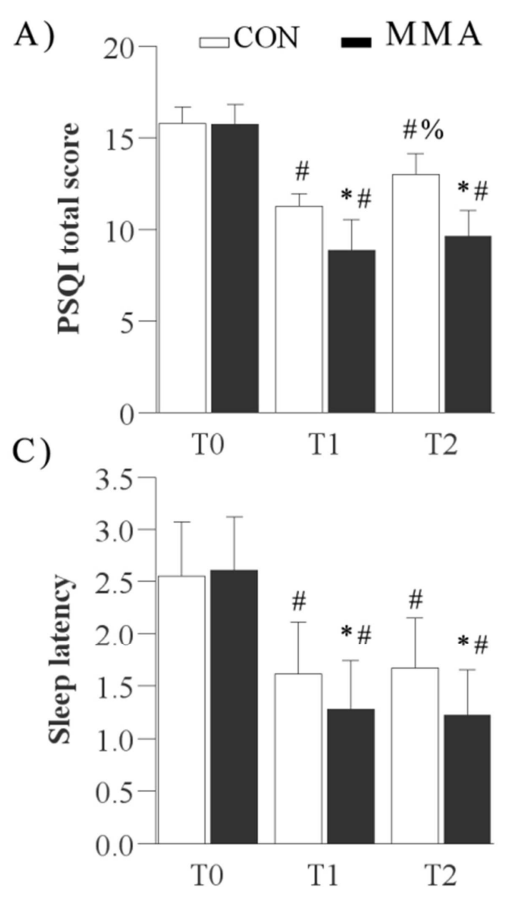

well as age as a covariate showed that the main effects of treatment, time, and age were significant, as summarized in Table 1. The significance of the interaction effects of treatment $\times$ time indicated significant effects of the MMA intervention on sleep quality in insomniac patients, as compared with the sole ANBR procedure $(p<0.001)$. Post hoc comparisons showed that the ANBR procedure in the Control Group had reduced the PSQI significantly at T1 compared with T0. Though the ANBR showed significant effects at T2 in the controls, the effect size decreased significantly at T2 compared with T1 (Figure 1A). MMA therapy showed significant effects at $\mathrm{T} 1$ as well as T2, as compared with T0. Furthermore, the effect size of MMA therapy was significantly greater than that of the ANBR procedure at time T1 $(t=9.59, p<0.001)$. Taken together, both ANBR and MMA improved patients' sleep quality. The effects of MMA on improving sleep quality were greater and more endurable compared with ANBR.

Table 1. Multivariate analysis results on PSQI global score. Treatment groups included the Control Group and the MMA Group; Time periods included T0, T1, and T2; Gender is a factor (male and female); Age is a covariate.

\begin{tabular}{lll}
\hline Effect & F Value & Pr $>$ F \\
\hline Treatment & 90.34 & $<.001$ \\
Time & 261.87 & $<.001$ \\
Treatment $\times$ Time & 22.46 & $<.001$ \\
Gender & 2.53 & 0.115 \\
Treatment $\times$ Gender & 0.56 & 0.456 \\
Gender $\times$ Time & 1.26 & 0.287 \\
Age & 8.63 & 0.004 \\
\hline
\end{tabular}

B )

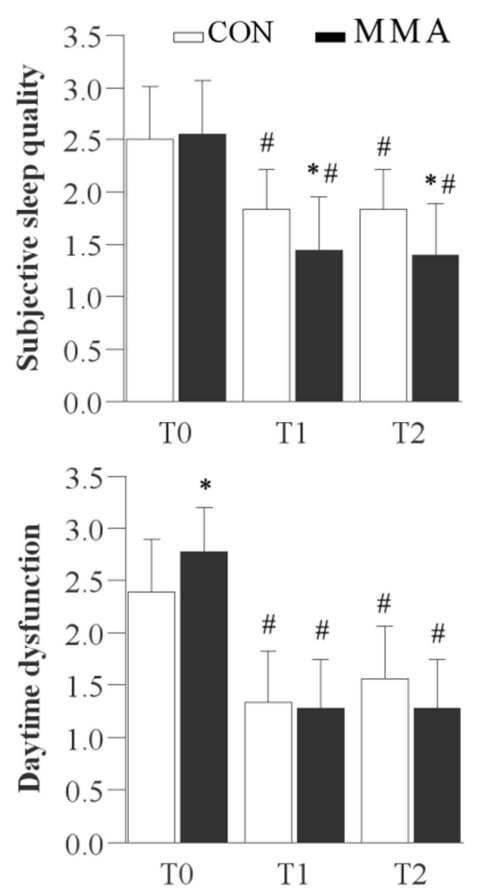

Figure 1. PSQI global scores as well as 3 component scores. Patients with primary insomnia were divided into two groups of Control Group (CON) and MMA treatment group (MMA), and were measured by PSQI prior to study involvement (T0), after 8-week treatment (T1), and at follow-up 4-week post-treatment (T2). A): PSQI global score; B): Subjective sleep quality; C): Sleep latency; D): Daytime dysfunction. " significant differences in MMA vs. CON at the same time point of measurement; " significant differences in measurements in the same treatment group at T1, T2 compared with T0. ${ }^{\circ}$ significant differences in measurements in the same treatment group at T2 compared with T1. Data were expressed as Mean \pm S.D. 


\subsection{Internal Homogeneity of the PSQI Measurements}

The consistency of the PSQI measurement was checked by the PROC CORR of SAS using Cronbach's alpha coefficient, as shown in Table 2. The six component scores (subjective sleep quality, sleep latency, sleep duration, habitual sleep efficiency, sleep disturbances, and daytime dysfunction) of the PSQI had an overall reliability coefficient of 0.89 (Cronbach's alpha coefficient), indicating a high degree of internal consistency. The largest component-total Pearson correlation coefficient was found for subjective habitual quality $(r=0.83, p<0.001)$, while the smallest was found for habitual sleep efficiency $(r=0.67, p<0.001)$. Taken together, the PSQI global score was internally consistent, and the six component scores appeared to measure a particular aspect of the same overall clinical construct, namely, sleep quality.

Table 2. Cronbach's alpha coefficients with deleted variable for the 6 components of PSQI.

\begin{tabular}{|c|c|c|c|c|}
\hline \multirow[t]{3}{*}{ Deleted Variable } & \multicolumn{2}{|c|}{ Raw Variables } & \multicolumn{2}{|c|}{ Standardized Variables } \\
\hline & Correlation & \multirow{2}{*}{ Alpha } & Correlation & \multirow{2}{*}{ Alpha } \\
\hline & with Total & & with Total & \\
\hline $\begin{array}{l}\text { Subjective sleep } \\
\text { quality }\end{array}$ & 0.76 & 0.87 & 0.76 & 0.87 \\
\hline Sleep latency & 0.77 & 0.86 & 0.78 & 0.86 \\
\hline Sleep duration & 0.65 & 0.88 & 0.65 & 0.89 \\
\hline $\begin{array}{l}\text { Habitual sleep } \\
\text { efficiency }\end{array}$ & 0.62 & 0.89 & 0.62 & 0.89 \\
\hline Sleep disturbances & 0.78 & 0.86 & 0.78 & 0.86 \\
\hline $\begin{array}{l}\text { Daytime } \\
\text { dysfunction }\end{array}$ & 0.71 & 0.87 & 0.71 & 0.88 \\
\hline
\end{tabular}

\subsection{Changes in the Six Components of the PSQI}

Multivariate analyses on the respective six components of the PSQI using treatment, gender, and time showed that three component scores of subject sleep quality, sleep latency, improved significantly in patients receiving MMA treatment compared with the control, as shown in Figure 1B, 1C. Although the component score of daytime dysfunction differed significantly at T0 in the MMA patients, MMA therapy significantly reduced the levels of daytime dysfunction as the ANBR procedure (Figure 1D). In contrast, MMA therapy did not show significant effects in the 3 component scores of sleep duration, habitual sleep efficiency, and sleep disturbances according to the multivariate analyses (data not shown).

\section{Discussion}

In summary, MMA therapy was quantitatively measured by an experimental design with each 18 patients diagnosed as primary insomnia in the Control and MMA groups, respectively. PSQI was adopted to measure the clinimetric properties of sleep disorder on three occasions of $\mathrm{T} 0, \mathrm{~T} 1$, and
T2. The control patients received ANBR procedure, while the MMA patients received MMA therapy apart from the ANBR procedure. The PSQI global scores decreased significantly in the patients receiving the additional MMA therapy. Further analyses on the 6 components of the PSQI showed that the improvements in sleep quality were attributable to the improvements of 3 components of subjective sleep quality, sleep latency, and daytime dysfunction. Cronbach's alpha coefficients showed that the improvements of sleep quality were consistent and reliable in patients receiving MMA therapy.

In this study, patients receiving ANBR were used as controls for three reasons. First, the ANBR is a physical therapy approved by the healthcare authorities, which dictates that patients with medical conditions seeking treatment should be treated by healthcare practitioners; therefore, blank control (i.e., no therapeutic treatment) was not feasible in clinical practice in the context of a tertiary teaching hospital. Second, the ANBR that is based on the theory of Heart Math [13] conforms to the view of insomnia by the traditional Mongolian medicine in that insomnia is caused by imbalance and/or dysfunction of the heart, liver, and kidney $[14,15]$. Although this holistic and metaphysical view on insomnia, characteristic of energy-flowing and meridians, is largely different from the evidence-based Western medicine, traditional Mongolian Medicine practitioners firmly believe that evidence-based modern science will be finding evidence for the key roles of heart and liver in maintaining homeostasis, as pioneered by Heart Math [12] Third, the ANBR system is contained in a SPCS system, from which biological data, such as blood pressure and heart rate, can be readily read; therefore, patients' receiving the ANBR procedure is an established practice in the Traditional Mongolian Department to achieve optimal patient outcome or prevention of adverse effects.

For practical reasons, study subjects were referred by the Department of Psychology and/or Internal Medicine, since insomniac patients tend to seek healthcare service in departments other than the Traditional Mongolian Medicine. Since publicly recruiting subjects for healthcare research involvement is not an established practice is China, sampling bias could be introduced when the study subjects were recruited by advertisements. In this study, the Pittsburgh Sleep Quality Index was adopted as the clinical construct in evaluating sleep disorders. Since one component was eliminated from the original 7-component PSQI, the actually scale of PSQI was $0-18$ in this study. The Cronbach's alpha coefficient was 0.89 in this study, which was higher than 0.83 reported by Buysse DJ. et al [9]. This difference was presumably due to the elimination of one component (component 6: use of sleeping medication) from the original 7-component PSQI. In our preliminary experiments, we tried polysomnography and sham acupuncture. Since this study was designed using outpatients as the subjects, their living environments had to be taken into account when conducting polysomnography. We suspected that more confounding 
factors (the acceptance of polysomnography from patients as well as their family members) may be introduced by polysomnography. We propose that polysomnography appears to be applicable for researches on insomnia using inpatients as study subjects. Since acupuncture is known to have significant placebo effects, sham acupuncture has been developed to address this methodological challenge [16]. We identified larger variation in outcome measures when applying of sham acupuncture (needle with retractable tip) in our preliminary experiments; therefore, we decide not to publish the data of sham acupuncture in this article. The uniqueness of the challenges in designing a Randomized Control Trial for acupuncture researches has been summarized in a recent review [17].

\section{Conclusion}

During the last decade, the researchers of Traditional Mongolian Medicine in our hospital have come to realize the importance of evidence-based Western medicine in investigating the effects of acupuncture in terms of neurology [17], autonomic activation by acupuncture [5], molecular biology, public health, experimental design, and statistical analysis. We are planning to address these issues in the future with respects to animal model, sham acupuncture techniques, as well as molecular biology.

\section{Acknowledgements}

This research is funded by the following projects:the Nature Science Foundation of Inner Mongolia Autonomous Region (2013MS1224); project of incentive funds for guidance of scientific and technical innovation in Inner Mongolia Autonomous Region-research on acupoints and operation technique standardization of Mongolian medicine warm needling therapy (No.: 2014cztcxyd); National Natural Science Foundation of China- research on the hypnotic role of Mongolian medicine warm needling for rats with insomnia caused by PCPA and its mechanism (No.: 81260571); public health project of the state administration of traditional Chinese medicine- research and promotion of Mongolian medicine-brain shaking therapy for treatment of cerebral concussion (No. gjzyyglj 11 agl nzd); public health project of the state administration of traditional Chinese medicinearrangement and annotation of literature on Mongolian medicine therapies (No.: gjzyyglj 11 agl); National Natural Science Foundation of China-Regulatory effects of Mongolian medical warm needle acupuncture on miRNAs and PAX8/APP protein in insomniac rats and mechanism study on insomnia treatment (No.: 81560801); Science and Technology Innovation Fund of Provincial Department of Finance, Inner Mongolia Autonomous Region; Collaborative Innovation Project of Mogolian Medicine, Inner Mongolia Autonomous Region; Technology Reserve Project of Provincial Department of Science and Technology, Inner Mongolia Autonomous Region.

\section{References}

[1] Katz DA and McHorney CA: The relationship between insomnia and health-related quality of life in patients with chronic illness. Journal of Family Practice 51: 229-236, 2002.

[2] Morin CM and Barlow DH: Insomnia: Psychological assessment and management. Guilford Press New York, 1993.

[3] Ota A, Masue T, Yasuda N, Tsutsumi A, Mino Y and Ohara H: Association between psychosocial job characteristics and insomnia: an investigation using two relevant job stress models - the demand-control-support (DCS) model and the effort-reward imbalance (ERI) model. Sleep medicine 6: 353-358, 2005.

[4] Morin CM, LeBlanc M, Daley M, Gregoire J and Merette C: Epidemiology of insomnia: prevalence, self-help treatments, consultations, and determinants of help-seeking behaviors. Sleep medicine 7: 123-130, 2006.

[5] Huang W, Kutner N and Bliwise DL: Autonomic activation in insomnia: the case for acupuncture. Journal of clinical sleep medicine: JCSM: official publication of the American Academy of Sleep Medicine 7: 95, 2011.

[6] Le-qin W: Observation on therapeutic effects of scraping therapy and warming acupuncture-moxibustion on 50 cases of fasciitis of back muscles [J]. Chinese Acupuncture \& Moxibustion 7: 008, 2006.

[7] Tao S, Qi G and Chao H: Clinical Study on Treatment of Hypercholesterolemia by Acupuncture with the Needle Warmed by Burning Cap Like Moxa Cone [J]. CHINESE ACUPONCTURE \& MOXIBUSTION 7: 1999.

[8] Zhangtao CW: Clinical observation of synthesis to treat 60 cases of insomnia. Nei Mongol Journal of Traditional Chinese Medicine 6: 006, 2010.

[9] Buysse DJ, Reynolds CF, Monk TH, Berman SR and Kupfer DJ: The Pittsburgh Sleep Quality Index: a new instrument for psychiatric practice and research. Psychiatry research 28: 193-213, 1989.

[10] Chen Y-F: Chinese classification of mental disorders (CCMD-3): towards integration in international classification. Psychopathology 35: 171-175, 2002.

[11] Roth T: Insomnia: definition, prevalence, etiology, and consequences. Journal of clinical sleep medicine: JCSM: official publication of the American Academy of Sleep Medicine 3: S7, 2007.

[12] Childre DL, Martin H and Beech D: The Heart Math Solution: The Institute of Heart Math's Revolutionary Program for Engaging the Power of the Heart's Intelligence. Harper One, 1999.

[13] Childre D and Rozman D: Transforming depression: The Heart Math solution to feeling overwhelmed, sad, and stressed. New Harbinger Publications, 2007.

[14] Guo J, Wang L-P, Liu C-Z, et al: Efficacy of acupuncture for primary insomnia: a randomized controlled clinical trial. Evidence-Based Complementary and Alternative Medicine 2013: 2013 
[15] Leach MJ and Page AT: Herbal medicine for insomnia: A systematic review and meta-analysis. Sleep medicine reviews 24: $1-12,2015$.

[16] Kaptchuk TJ, Stason WB, Davis RB, et al: Sham device v inert pill: randomised controlled trial of two placebo treatments. Bmj 332: 391-397, 2006.
[17] Yeung W-F, Chung K-F, Leung Y-K, Zhang S-P and Law AC: Traditional needle acupuncture treatment for insomnia: a systematic review of randomized controlled trials. Sleep medicine 10: 694-704, 2009.

[18] White A, Cummings TM and Filshie J: An introduction to western medical acupuncture. Churchill Livingstone/Elsevier Edinburgh, 2008. 